\title{
Tiempos de llegada al hospital y hasta la atención neurológica de pacientes con ictus agudo. Análisis de un registro multicéntrico nacional: registro EPICES (II)
}

\author{
José Castillo, José Vivancos-Mora, en nombre de los investigadores del registro EPICES
}

Introducción. Los tiempos en la llegada al hospital o el retraso hospitalario en la atención especializada a los pacientes
con ictus siguen siendo la primera causa para no recibir el mejor de los tratamientos posibles.

Objetivo. Analizar los tiempos de llegada al hospital y hasta la atención neurológica en una serie multicéntrica de pacientes con ictus.

Pacientes y métodos. El registro EPICES es un estudio observacional y prospectivo realizado con metodología de registro de historias clínicas consecutivas en pacientes con ictus hospitalizados bajo atención neurológica. La muestra para analizar los tiempos inicio-puerta fue de 5.454 pacientes, para el tiempo puerta-neurólogo fue de 5.379 pacientes y para el tiempo de permanencia en urgencias fue de 5.825 pacientes.

Resultados. El 90\% de los pacientes presentó el ictus entre las 8 y las 24 horas. La estación del año no influyó en la frecuencia, ni en la distribución de los distintos tipos de ictus. El tiempo inicio-puerta fue de $264,1 \pm 318,3$ minutos; el 34,7\% de los pacientes acudió al hospital en la primera hora después del inicio de los síntomas y el 63,7\% en las tres primeras horas. El tiempo puerta-neurólogo fue de 378,1 $\pm 731,4$ minutos; la edad del paciente $(p<0,0001)$ y el antecedente de un ictus previo $(p<0,0001)$ alargaron significativamente este tiempo. La existencia de guardias de neurología y de equipos de ictus se asoció a una disminución significativa de los tiempos intrahospitalarios.

Conclusión. En relación con datos previos, el estudio actual muestra una reducción importante de los tiempos iniciopuerta y puerta-neurólogo en nuestro medio.

Palabras clave. Estructuras asistenciales. Ictus. Tiempos asistenciales. Tiempos extrahospitalarios. Tiempos intrahospitalarios.

\section{Introducción}

Con una década de retraso en relación con el infarto agudo de miocardio, el ictus se considera hoy una indiscutible emergencia neurológica. Este retraso en la consideración emergente del ictus se puede deber a la diferencia de nueve años entre la publicación de la eficacia del tratamiento trombolítico en el infarto de miocardio (1986) [1] y en el ictus (1995) [2]. Otro factor sin duda importante es la atención casi exclusivamente cardiológica del infarto agudo de miocardio en contraposición con la atención multidisciplinar del ictus por un colectivo variado de profesionales, como neurólogos, radiólogos, internistas, intensivistas y geriatras, muchos de ellos con escasa experiencia en el tratamiento de procesos emergentes [3].

A pesar de todas las dificultades, el ictus ha alcanzado la consideración de una emergencia neurológica, y el retraso en su atención repercute en un incremento de la lesión cerebral, peor pronóstico, pérdida de la eficacia de los cuidados sanitarios y en un aumento de las complicaciones evolutivas y terapéuticas [4-6]. Sin embargo, los tiempos en la llegada al hospital o el retraso hospitalario en la atención especializada siguen siendo una de las primeras causas para no recibir el mejor de los tratamientos posibles [7].

Entre enero y junio de 1994, 18 unidades neurológicas de otros tantos hospitales del sistema público de salud español analizaron los tiempos de latencia en la atención de 794 pacientes con ictus. El 32,1\% de los pacientes llegó a urgencias en las tres primeras horas desde el inicio de los síntomas o del último momento libre de síntomas en los ictus al despertar (el 58,2\% en las seis primeras horas), pero sólo el 9,6\% recibió atención neurológica (el 22,7\% en seis horas) [8]. Ningún otro estudio multicéntrico de ámbito nacional español ha actualizado estos datos.

Un reciente y extenso análisis ha recopilado los datos de 75 estudios y de 183.009 pacientes, y ha comprobado una disminución del tiempo de llega-
Servicio de Neurología; Hospital Clínico Universitario de Santiago; Universidad de Santiago de Compostela; Santiago de Compostela, A Coruña (J. Castillo) Servicio de Neurología; Instituto de Investigación Sanitaria Princesa; Hospital Universitario La Princesa; Madrid, España (J. Vivancos-Mora).

Correspondencia:

Dr. José Castillo Sánchez. Servicio de Neurología. Hospital Clínico Universitario de Santiago. Travesa da Choupana, s/n. E-15706 Santiago de Compostela (A Coruña).

E-mail:

jose.castillo@usc.es

Declaración de intereses: El registro EPICES fue patrocinado por Pfizer España. J.C. y J.V.M. recibieron honorarios por la dirección del registro.

Aceptado tras revisión externa: 19.10.11.

Cómo citar este artículo: Castillo J, Vivancos-Mora J, en nombre de los investigadores del registro EPICES. Tiempos de llegada al hospital y hasta la atención neurológica de pacientes con ictus agudo. Análisis de un registro multicéntrico nacional: registro EPICES (II). Rev Neurol 2012; 54: 461-7.

Investigadores participantes en el registro EPICES:

J. Abella-Corral, M.J. Abenza-Abildua, M. Alonso de Leciñana, J. ÁlvarezGutiérrez, J. Álvarez-Sabín, C. AntónGonzález, J. Arenillas-Lara, M. AriasRodríguez, A. Arjona-Padillo,

C. Barrero-Ramírez, A. BarrosoMerinero, L. Benavente-Fernández, L. Berenguer-Ruiz, Y. BravoAnguiano, D. Cánovas-Verge, P. Carbonell-Corvillo, A.M. CarraVélez, I. Casado-Menéndez, I. Casado-Naranjo, L. Castilla-Guerra, J. Castillo-Sánchez, C. Cid-Rodríguez, M.J. Cruz-Huertas, P. de JuanHernández, C. Díaz-Marín, F. DíazOtero, E. Díez-Tejedor, F. DomínguezSanz, J. Egido-Herrero, M.I. Escalza-Cortina, E. Escolar-Escamilla, 
J. Estela-Herrero, J. Fernández-Ferro, E. Franquet-Gómez, R. GalianoBlancart, J. Gallego-Cullere, J.C. García-Moncó, A. García-Pastor L. García-Tuñón, A. Gil-Núñez, J.C. Giner-Bernabeu, S. GomaraLópez, A. Gómez Díaz-Castroverde, J.C. Gómez-Sánchez, C. GómezEscalonilla, C. González-Rodríguez, J. Gutiérrez-García, R. HernándezClares, J.J. Hernández-Regadera, L. Hernández-Rubio, M.E. HerreroPrieto, G. Izquierdo-Ayuso M.C. Jiménez-Martínez, M. JiménezNieto, M. Lezcano-Rodas, J.I. LópezGastón, J.F. Maestre-Moreno, C.G. Marrero-Falcón, J. Martí-Fábregas, S. Martín-Balbuena, M.M. MartínezMartínez, E. Martínez-Vila, A. MartínezYélamos, J. Masjuán-Pallejo, V. MecaLallana, J.P. Medina-Báez, A. Medina-

Rodríguez, M.V. Mejías-Olmedo,

E. Meneu-García, M. Millán-Torne

C. Monforte-Dupret, A. Moral-

Pijaume, J. Muñoz-Torrero Rodríguez,

C. Naranjo-Fernández, J.J. OchoaSepúlveda, J. Oliva-Navarro,

E. Orts-Castro, E. Palacio-Portilla,

E. Palomeras-Soler, R. Pego-Reigosa,

M.C. Peinado-Cantero, J. Peña-

Martínez, C. Pérez, N. Pérez de la

Ossa-Herrero, C. Pérez-Lázaro, R.

Piñeiro-Bolaño, M.A. Pons-García,

A. Ponz de Tienda, F. PurroyGarcía, J.M. Ramírez-Moreno,

M. Rebollo Álvarez-Amandi, A. Rey

Pérez, G. Ruiz-Ares, J. Sanahuja-

Montesinos, O. Sánchez del Valle

J. Sánchez-Herrero, C. SánchezSánchez, J. Saura-Salvado, T. Segura-

Martán, M. Seijo-Martínez, M.C.

Serrano-Munuera, M. Serrano-Ponz,

J. Tejada-García, T. Tortosa-Sánchez,

J. Trejo-Gabriel y Galán, P. Vázquez-

Alen, I. Villegas-Martínez, J. VivancosMora, A. Yusta-Izquierdo.

(c) 2012 Revista de Neurología

Tabla I. Tiempos inicio-puerta, puerta-neurólogo y de permanencia en urgencias en diversas situaciones (en minutos).

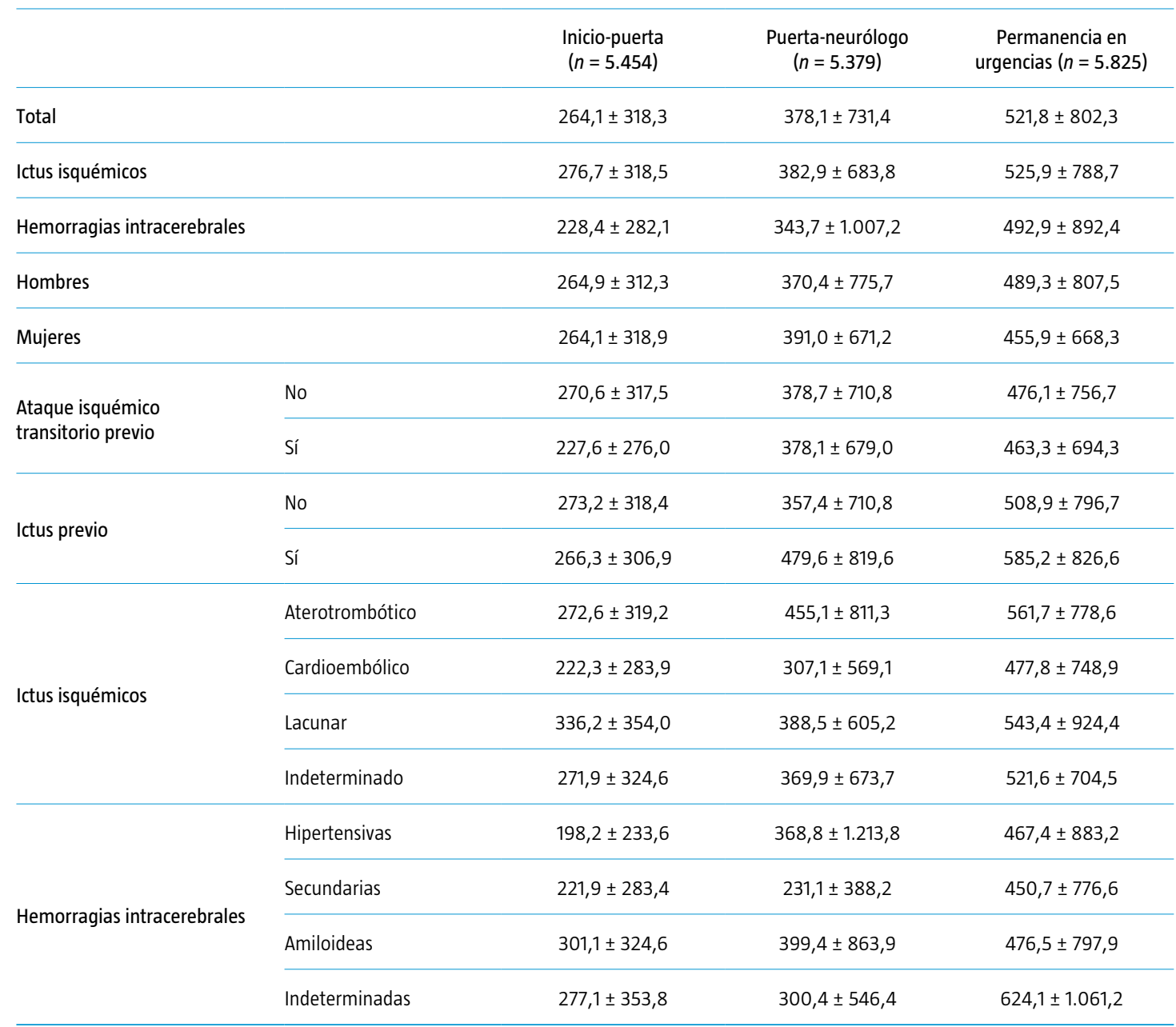

da al hospital de un 6\% anual; sin embargo, el tiempo entre la llegada al hospital y la atención por el personal de urgencias no se ha modificado desde el año 1990 hasta el 2008 [9].

Este trabajo expone los resultados del análisis de los tiempos de llegada al hospital y hasta la atención neurológica de los pacientes incluidos en el registro EPICES (Epidemiología del Ictus en España).

\section{Pacientes y métodos}

El análisis de los tiempos de llegada al hospital (inicio-puerta) y el tiempo hasta la atención neurológica (puerta-neurólogo) constituyó uno de los objetivos secundarios del registro EPICES. La descripción general y metodología del registro EPICES ya se ha publicado [10]; en resumen, se trata de un registro observacional y prospectivo realizado con metodología de registro de historias clínicas consecutivas en pacientes con ictus hospitalizados bajo atención neurológica. La recogida de datos por parte del investigador incluyó a la totalidad de los pacientes ingresados en cuatro meses completos (abril, junio y septiembre del 2008 y enero del 2009) para estudiar la estacionalidad. Se incluyó la totalidad de las historias clínicas de los pacientes ingresados y con diagnóstico de ictus. Se excluyeron los casos de ataque isquémico transitorio, hemorragia subaracnoidea, hemorragias cerebrales traumáticas, hematomas epidurales y hematomas subdurales.

Se registró la hora del inicio de los síntomas referida por el paciente o por un familiar; en los casos de ictus al despertar se anotó la hora en que por úl- 
tima vez se confirmó que el paciente estaba asintomático. La hora de llegada al hospital (puerta) se recogió del registro informático del ingreso. El tiempo hasta la atención neurológica fue obtenido por el responsable del estudio, de la misma forma que el tiempo de permanencia en el servicio de urgencias.

Para la definición de equipos y unidades de ictus se utilizaron los criterios del Grupo de Estudio de Enfermedades Cerebrovasculares de la Sociedad Española de Neurología [11]. Se consideró la existencia de guardias de neurología tanto en el caso de guardias requeridas o de presencia física, durante 12 o 24 horas.

\section{Análisis estadístico}

La población utilizada para el análisis estadístico incluyó a todos los pacientes que cumplieron todos los criterios de inclusión y ninguno de los criterios de exclusión. El tipo de distribución de las variables cuantitativas se evaluó mediante el test de Kolmogorov-Smirnov. Para el análisis descriptivo se utilizaron medias \pm desviaciones estándares o medianas (percentil 25, percentil 75) según la distribución normal o no de las variables; las variables cualitativas se expresaron como porcentajes. La comparación entre variables cualitativas se llevó a cabo mediante el test de $\chi^{2}$, y para las cuantitativas, el test de la $t$ de Student o de Mann-Whitney, según su distribución. La comparación entre diversas variables cuantitativas se realizó mediante un análisis de la varianza. Las correlaciones entre variables se hicieron aplicando el coeficiente de Pearson o de Spearman, según su distribución. Para efectuar el análisis se utilizó el programa estadístico SPSS v. 16.0.

\section{Resultados}

De los 6.197 pacientes válidos para el análisis de la variable principal, en 743 no se registró el tiempo inicio-puerta, en 818 el tiempo puerta-neurólogo y en 372 el tiempo de permanencia en urgencias. En total, la muestra para analizar los tiempos iniciopuerta fue de 5.454 pacientes, para los tiempos puerta-neurólogo fue de 5.379 pacientes y para los tiempos de permanencia en urgencias fue de 5.825 pacientes.

La estación del año no influyó en la presentación de ictus isquémicos o hemorrágicos $(p=0,493)$, ni en los distintos tipos de ictus isquémicos $(p=0,353)$, ni en los diferentes tipos de hemorragias intracerebrales $(p=0,083)$.
Figura 1. Porcentaje de pacientes con ictus isquémico y hemorragia cerebral según la hora de inicio de la sintomatología. La hora de inicio de los pacientes con ictus al despertar es la última hora en la que se encontraron asintomáticos.

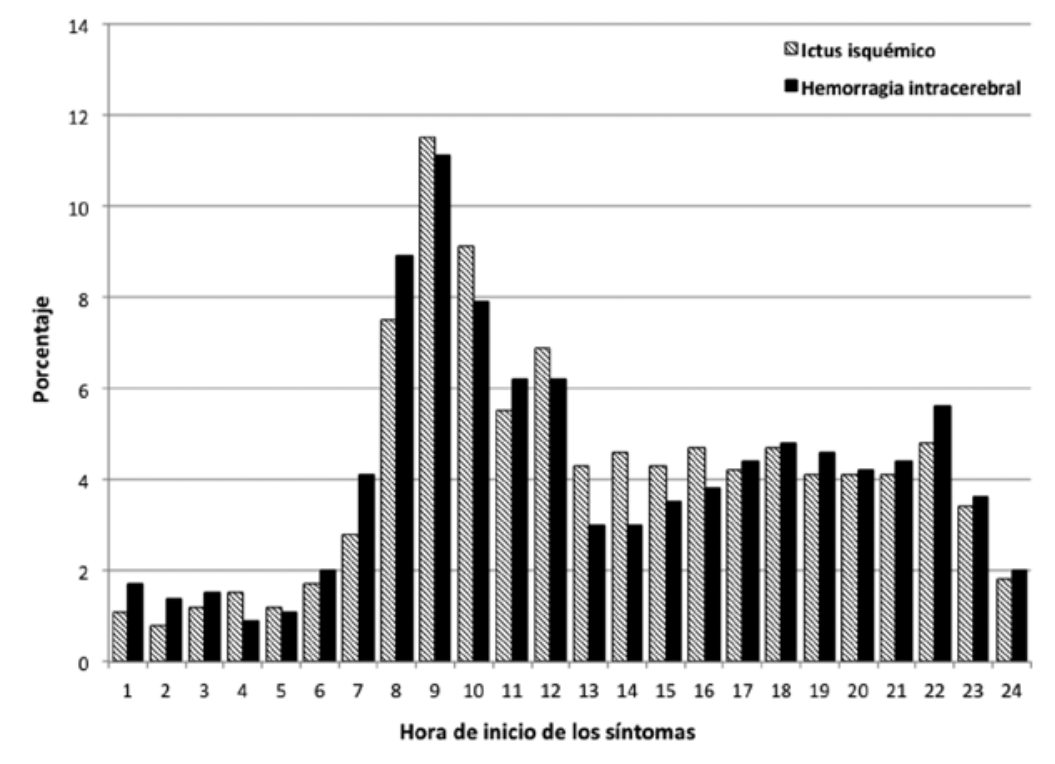

El 53,3\% de los pacientes presentó el ictus entre las 8 y las 15 horas, el 36,0\% lo sufrió entre las 15 y las 24 horas, y el 10,7\% entre las 0 y las 8 horas. Estos resultados incluyen a 1.035 pacientes $(19,0 \%)$ que presentaron un ictus al despertar. La distribución de la hora de inicio del cuadro clínico se expresa en la figura 1. La hora de inicio fue similar en la isquemia y la hemorragia cerebral $(p=0,408)$, entre los distintos tipos de ictus isquémicos $(p=0,184) \mathrm{y}$ de hemorragias cerebrales $(p=0,532)$.

En el subgrupo de pacientes que presentó un ictus isquémico al despertar, el porcentaje fue similar en los diversos tipos (un 25,7\% aterotrombótico, un $23,2 \%$ cardioembólico, un $24,8 \%$ lacunar y un $23,9 \%$ de origen indeterminado; $p=0,227)$. Solamente el $1,3 \%$ de las hemorragias intracerebrales se presentó en el momento del despertar.

El tiempo inicio-puerta fue de 264,1 $\pm 318,3 \mathrm{mi}$ nutos, superior en los isquémicos que en las hemorragias $(p<0,0001)$ (Tabla I). El 34,7\% de los pacientes acudió al hospital en la primera hora después del inicio de los síntomas, el $63,7 \%$ en las tres primeras horas y el 78,1\% en las seis primeras horas (Fig. 2). En los ictus isquémicos, el tiempo iniciopuerta fue menor en los cardioembólicos que en los aterotrombóticos e indeterminados $(p<0,0001)$, y resultó significativamente mayor en los lacunares 
Figura 2. Tiempos inicio-puerta y puerta-neurólogos expresados como porcentaje de pacientes por cada fracción horaria.

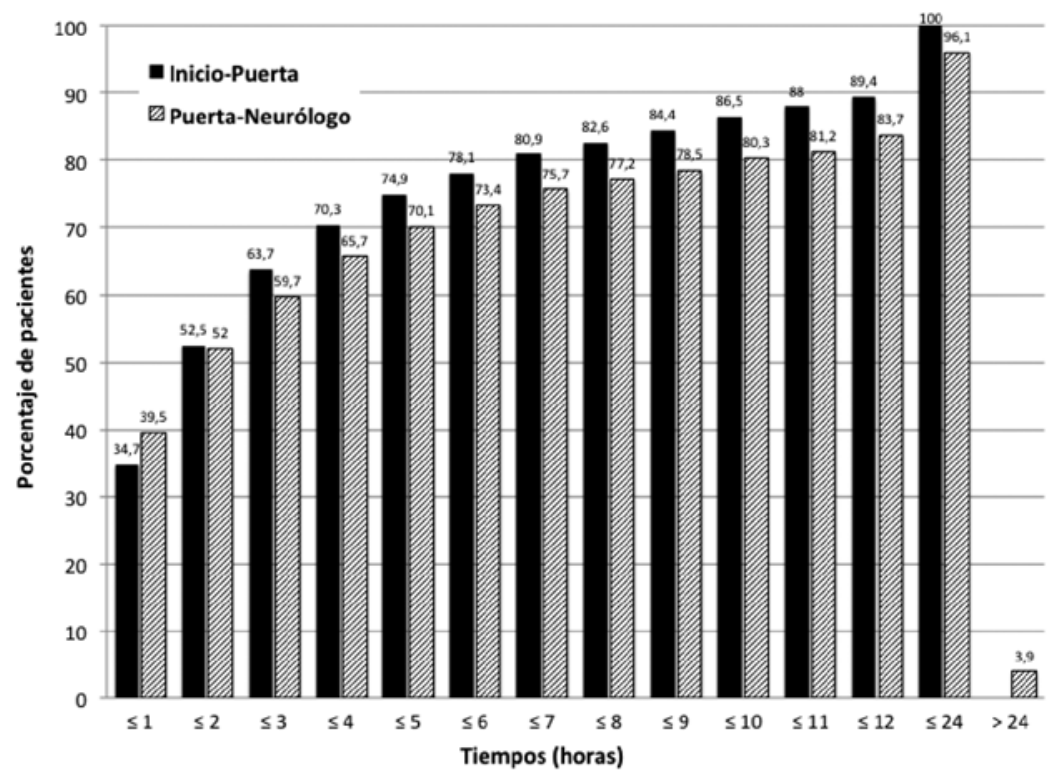

Figura 3. Distribución de los tiempos puerta-neurólogos en relación con la edad de los pacientes. Puede apreciarse que el tiempo de atención especializada se retrasa en los pacientes de mayor edad.

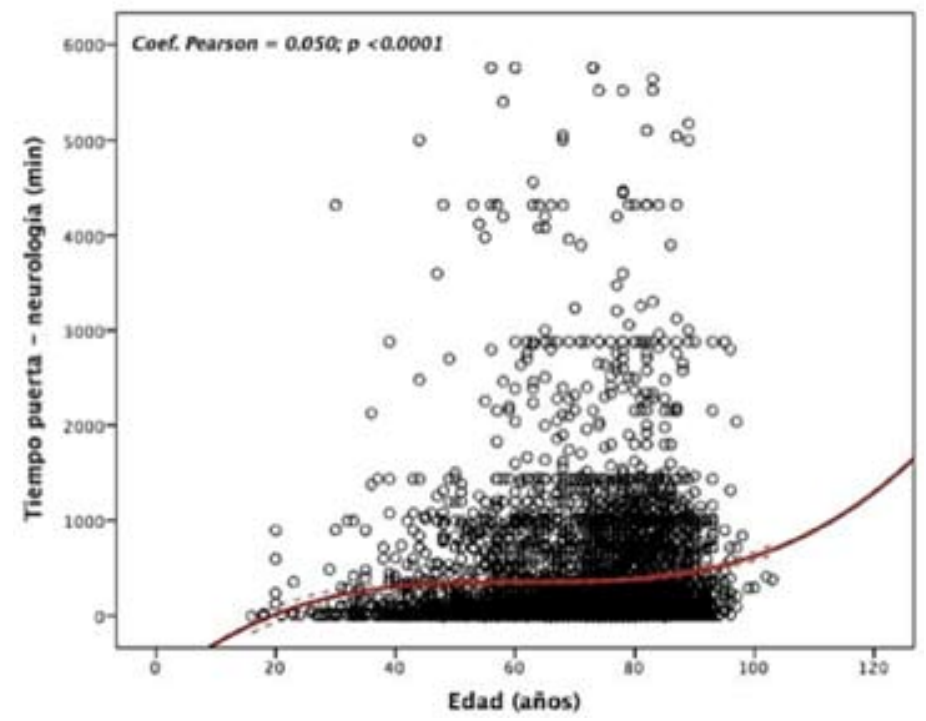

$(p<0,0001)$. En las hemorragias intracerebrales, las hipertensivas y las secundarias llegaron antes que las amiloideas e indeterminadas $(p=0,002)$.
Los tiempos inicio-puerta no se vieron influidos por el sexo $(p=0,453)$, pero sí por la edad (coeficiente de Pearson $=-0,038 ; p=0,005)$; a mayor edad los pacientes acudieron antes al hospital. El antecedente de ataques isquémicos transitorios previos $(p<0,0001)$, pero no de un ictus previo $(p=0,304)$, condicionó un menor tiempo inicio-puerta.

El tiempo puerta-neurólogo fue de 378,1 \pm 731,4 minutos, similar en los ictus isquémicos y en las hemorragias intracerebrales $(p=0,492)$. El 39,5\% de los pacientes con ictus que acudieron a urgencias recibió atención neurológica en la primera hora; sin embargo, un $26,6 \%$ de los pacientes no fue atendido por un neurólogo en las seis horas desde la llegada al hospital y un 16,3\% tampoco en las 12 primeras horas (Fig. 2). En los ictus isquémicos el tiempo puerta-neurólogo fue menor en los infartos cardioembólicos $(p<0,0001)$ que en el resto de los tipos etiológicos; este tiempo fue similar en los diferentes tipos de hemorragias intracerebrales (Tabla I). Ni el sexo $(p=0,606)$ ni el antecedente de ataque isquémico transitorio $(p=0,889)$ condicionaron una variación del tiempo puerta-neurólogo, pero sí el antecedente de ictus previo, que alargó significativamente este tiempo $(p<0,0001)$, así como la edad del paciente $(p<0,0001)$ (Fig. 3; tabla I).

El tiempo de permanencia en urgencias fue de $521,8 \pm 802,3$ minutos, similar entre la isquemia y hemorragia cerebral $(p=0,541)$, entre los distintos tipos de ictus isquémicos $(p=0,069)$ y de hemorragias cerebrales $(p=0,365)$. También fue independiente de la edad $(p=0,065)$, del sexo $(p=0,075)$ y de los antecedentes de ataque isquémico transitorio $(p=0,065)$. Los pacientes con ictus previos tuvieron una estancia en urgencias más prolongada $(p=$ 0,037) (Tabla I).

De los 66 centros que participaron en el registro EPICES, 19 tienen guardias de neurología $(28,8 \%)$, 17 disponen de unidades de ictus $(25,7 \%)$ y 35 manifestaron estar organizados como equipos de ictus $(50,1 \%)$. Los centros con guardias de neurología ingresaron a 2.153 pacientes $(34,7 \%)$, los centros con unidades de ictus ingresaron a 2.008 pacientes $(32,4 \%)$ y los centros con equipos de ictus ingresaron a 3.651 pacientes $(58,9 \%)$. Sólo el $37,7 \%$ de los pacientes ingresados en una unidad de ictus tenía guardia de neurología en la puerta; sin embargo, el porcentaje de pacientes ingresados por equipos de ictus con guardia de neurología en la puerta fue significativamente mayor (46,6\%; $p<0,0001)$. El 16,6\% de los pacientes incluidos en el registro EPICES $(n=1.027)$ fueron atendidos por neurólogos en centros sin guardia de neurología, unidades de ictus o equipos de ictus. 
El tiempo inicio-puerta fue inferior $(p<0,0001)$ en los centros con guardias de neurología $(229,7 \pm$ 296,3 minutos); la presencia de unidades de ictus o de equipos de ictus no condicionó la duración de este tiempo $(293,4 \pm 334,8$ frente a $286,4 \pm 328,9$ minutos; $p=0,682$ ). Todas las estructuras asistenciales para la atención a los pacientes con ictus consiguieron una disminución significativa de los tiempos puerta-atención neurológica (Tabla II). En la figura 4 se expresa el porcentaje de pacientes atendidos por los neurólogos en las seis primeras horas, y se puede comprobar que la existencia de guardias de neurología y de equipos multidisciplinares de ictus constituyen la estructura asistencial que permite atender más rápidamente a los pacientes con ictus agudos. El tiempo de permanencia en los servicios de urgencias en los centros con urgencias de neurología (345,4 $\pm 666,9$ minutos) es menor ( $p=$ $0,028)$ que en los centros con equipos de ictus $(436,1$ $\pm 706,3$ minutos) y que en los centros con unidades de ictus (504,7 $\pm 793,1$ minutos; $p<0,0001)$.

\section{Discusión}

El registro EPICES presenta resultados epidemiológicos limitados a los pacientes atendidos por neurólogos durante el período de hospitalización. Esto plantea el sesgo de no registrar a la totalidad de los pacientes con ictus atendidos por los 66 hospitales públicos españoles. Sin embargo, el desarrollo asistencial en nuestro país ha conseguido centrar en los servicios o unidades de neurología la mayor parte de la atención de los pacientes con ictus; el $82 \%$ de los pacientes con ictus en los centros que han participado en el registro EPICES (datos no mostrados) ha sido atendido por neurólogos.

En el registro EPICES no se han demostrado variaciones estacionales en el ingreso de pacientes con ictus isquémicos o hemorrágicos. Parece existir un incremento en la mortalidad (no en la incidencia del ictus) en relación con olas de calor, especialmente en la población más anciana (deshidratación, insuficiencia respiratoria, insuficiencia renal, etc.) [12-14], aunque otros estudios no han logrado confirmarlo [15]. Factores ambientales asociados con determinadas estaciones del año (como la presión atmosférica, el grado de humedad, la polución ambiental y otros) también han confirmado su asociación con la incidencia de ictus, pero la validez de estos estudios suele estar limitada a espacios geográficos muy localizados $[16,17]$. Un reciente estudio sobre el ingreso de ictus en 155 hospitales de Estados Unidos, realizado durante un período de cuatro años (2004-
Figura 4. Porcentaje de pacientes atendidos por el neurólogo en la primera, tercera y sexta hora desde la entrada en el hospital. El: equipo de ictus; GN: guardias de neurología; GN + El: guardias de neurología con equipo de ictus; $\mathrm{GN}+\mathrm{UI}$ : guardias de neurología con unidad de ictus; UI: unidad de ictus.

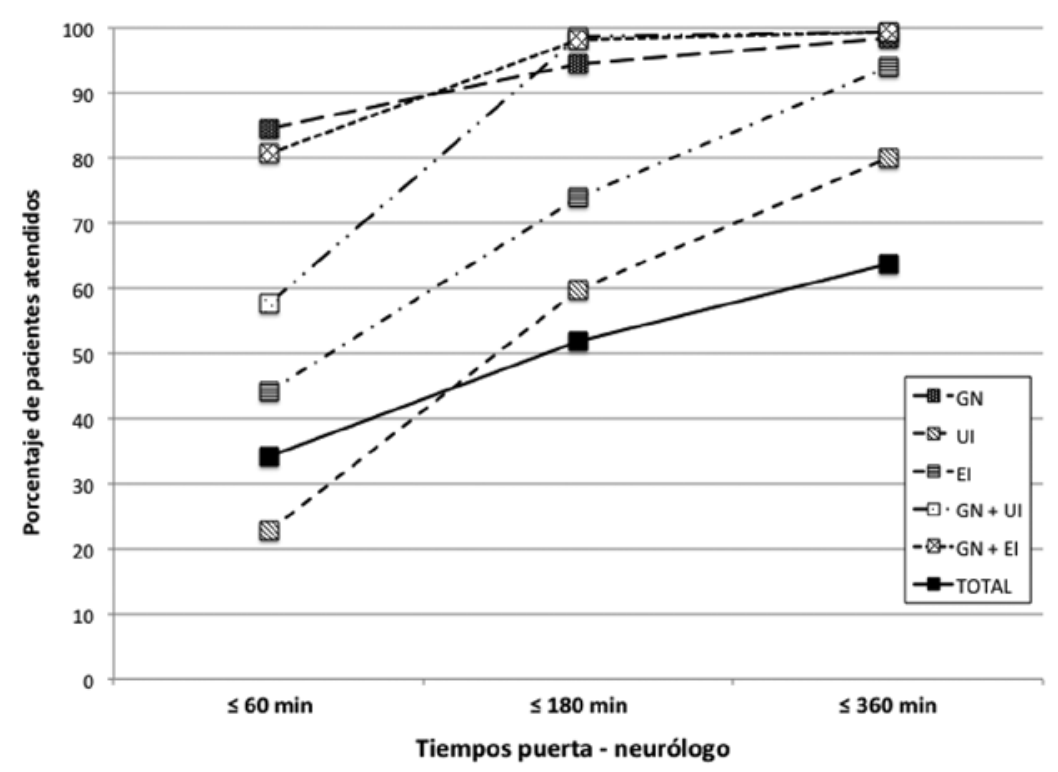

Tabla II. Tiempos puerta-neurólogo (en minutos) en relación con la estructura asistencial.

\begin{tabular}{lccc}
\hline & Sí & No & $p$ \\
\hline Guardias de neurología $(n=2.153)$ & $34,5 \pm 62,9$ & $607,3 \pm 871,1$ & $<0,0001$ \\
\hline Unidades de ictus $(n=2.008)$ & $227,8 \pm 207,6$ & $467,6 \pm 898,5$ & $<0,0001$ \\
\hline Equipos de ictus $(n=3.651)$ & $129,6 \pm 122,7$ & $1.285,2 \pm 1.123,1$ & $<0,0001$ \\
\hline Guardias + unidades de ictus $(n=757)$ & $66,8 \pm 31,7$ & $786,1 \pm 1.062,9$ & $<0,0001$ \\
\hline Guardias + equipos de ictus $(n=1.703)$ & $40,3 \pm 29,5$ & $1.284,9 \pm 1.124,1$ & $<0,0001$ \\
\hline
\end{tabular}

2008), ha registrado 196.439 ictus (98.930 isquémicos y 18.960 hemorrágicos) y no ha demostrado ninguna relación con la estación del año [18].

En el registro EPICES el 53\% de los pacientes presentó el ictus entre las 8 y las 15 horas y el $36 \%$ entre las 15 y las 24 horas; solamente en un $11 \%$ la sintomatología se inició entre las 0 y las 8 horas. Estos datos deben tener utilidad para la programación de las guardias de neurología (de 24 horas, o localizada, a partir de las 22 horas). Datos similares ya fueron comunicados por grupos españoles [19-21] 
y en un metaanálisis que incluyó 31 publicaciones y 11.816 ictus; en este estudio, el 55\% de los pacientes presentó el ictus entre las 6 y las 12 horas [22]. Sin embargo, todas estas referencias, incluidas las del registro EPICES, muestran la limitación que representa la arbitraria definición de los ictus al despertar, que se incluyen con un margen de tiempo habitualmente superior a las seis horas [23].

Es indudable que el conocimiento de las enfermedades cerebrovasculares (especialmente el ictus) en la población española es cada vez mejor, aunque los resultados conseguidos todavía no sean satisfactorios [24], por lo que su divulgación sigue siendo una obligación de los neurólogos. Este mejor conocimiento, la normalización del tratamiento trombolítico y el cambio de actitud de muchos médicos de atención primaria y de los servicios de emergencias extrahospitalarias son, sin duda, responsables de una más rápida llegada de los pacientes al hospital. En 14 años, el porcentaje de pacientes con ictus que acuden a un hospital público en España en la primera hora desde el inicio de los síntomas ha pasado del 10,2\% [8] al 34,7\%, lo que supone un incremento superior al $300 \%$. Esta diferencia se mantiene en el porcentaje de pacientes que llegan en las tres primeras horas (del 32,1 al 63,7\%) y en las seis primeras horas (del 58,2 al 78,1\%). Los tiempos de llegada por un ictus al hospital se han reducido en un $6 \%$ por año en un metaanálisis de 75 estudios desde el año 1990 hasta el 2008. La introducción del tratamiento trombolítico ha resultado ser un factor decisivo [9].

Otro dato positivo del registro EPICES ha sido la demostración de un acortamiento de los tiempos intrahospitalarios de atención a los pacientes con ictus, especialmente para la atención neurológica. En la primera hora desde la llegada al hospital la atención neurológica cubrió al 39,5\% de los pacientes, frente al 2,7\% en el año 1994 [8]. Estos resultados positivos españoles contrastan con un lamentable incremento de un $10,2 \%$ por año en el retraso de la atención neurológica en muchos estudios de países de varios continentes [9].

Sin embargo, un aspecto preocupante recogido en el registro EPICES es la disminución de la prioridad en la atención neurológica en los pacientes con ictus previos, y especialmente en los pacientes con mayor edad. La eficacia del tratamiento trombolítico y de los emergentes procedimientos intervencionistas puede desviar la atención del neurólogo hacia unos pocos pacientes y hacer que se olvide de la utilidad demostrada para la mayoría de la aplicación lo más precoz posible de los mejores cuidados neurológicos [25].
En conclusión, en este registro se demuestra que la implementación de vías o estructuras extra e intrahospitalarias consiguen disminuir los tiempos y facilitan la atención más precoz a los pacientes. La utilidad de las unidades de ictus sigue siendo incuestionable y es la mejor opción para los pacientes [26]; sin embargo, resulta difícil lograr un adecuado funcionamiento de las unidades de ictus en ausencia de guardias de neurología y de un equipo multidisciplinar en el hospital. En el registro EPICES se constata que la mitad de las unidades de ictus no dispone de guardias de neurología, y que todavía un $16,6 \%$ de los pacientes registrados fueron atendidos por neurólogos en hospitales sin guardia de neurología, unidades de ictus o equipos de ictus. La asociación de guardias de neurología y de un equipo multidisciplinar, más que las unidades de ictus aisladas, constituye la mejor estrategia para facilitar el más rápido y adecuado tratamiento a los pacientes con ictus agudos [27].

Bibliografía

1. Gruppo Italiano per lo Studio della Strepchinasi nell'Infarto Miocardio (GISI). Effectiveness of intravenous thrombolytic treatment in acute myocardial infarction. Lancet 1986; 1: 397-401.

2. National Institute of Neurological Disorders and Stroke rt-PA Stroke Study Group. Tissue plasminogen activator for acute ischemic stroke. N Engl J Med 1995; 333: 1581-7.

3. Herlitz J, Wireklinsundström B, Bang A, Berglund A, Svensson L, Blomstrand C. Early identification and delay to treatment in myocardial infarction and stroke: differences and similarities. Scand J Trauma Resusc Emerg Med 2010; 18: 48.

4. Ringelstein FB, Biniek R, Weiller C, Amneling B, Nolte PN, Thron A. Type and extent of hemispheric brain infarctions and clinical outcome in early and delayed middle cerebral artery recanalization. Neurology 1992; 42: 289-98.

5. Ueda T, Hatakeyama T, Kumon Y, Sakaki S, Uraoka T Evaluation of risk of hemorrhagic transformation in local intra-arterial thrombolysis in acute ischemic stroke by initial SPECT. Stroke 1994; 25: 298-303.

6. Dávalos A, Castillo J, Martínez-Vila E; for the Cerebrovascular Diseases Study Group of the Spanish Society of Neurology. Delay in neurological attention and stroke outcome. Stroke 1995; 26: 2233-7.

7. Cocho D, Belvís R, Martí-Fábregas J, Molina-Porcel L, DíazManera J, Aleu A, et al. Reasons for exclusion from thrombolitic therapy following acute ischemic stroke. Neurology 2005; 64: 719-20.

8. Castillo J, Dávalos A, Martínez-Vila E; por el Grupo de Estudio de las Enfermedades Cerebrovasculares de la Sociedad Española de Neurología. Tiempos de latencia en la atención de los pacientes con enfermedades cerebrovasculares agudas. Estudio multicéntrico. Rev Neurol 1996; 24: 427-30.

9. Evenson KR, Foraker R, Morris DL, Rosamond WD. A comprehensive review of prehospital and in-hospital delay times in acute stroke care. Inr J Stroke 2009; 4: 187-99.

10. Arias S, Vivancos-Mora J, Castillo J; en nombre de los investigadores del registro EPICES. Epidemiología de los subtipos de ictus en pacientes hospitalizados atendidos por neurólogos: resultados del registro EPICES (I). Rev Neurol 2012; 54: 385-93.

11. Álvarez-Sabín J, Alonso de Leciñana M, Gállego J, Casado I, Castillo J, Díez-Tejedor E, et al; Grupo de Estudio de 
Enfermedades Cerebrovasculares de la Sociedad Española de Neurología. Plan de atención sanitaria al ictus. Neurología 2006; 21: 717-26.

12. Rothwell PM, Wroe SJ, Stattery J, Warlow CP. Is stroke incidence related to season or temperature? The Oxfordshire Community Stroke Project. Lancet 1996; 347: 934-6.

13. Mastrangelo G, Fedeli U, Visentin C, Milan G, Fadda E, Spolaore P. Pattern and determinants of hospitalization during heat waves: an ecologic study. BMC Public Health 2007; 7: 200.

14. Metzger KB, Ito K, Matle TD. Summer heat and mortality in New York City: how hot is too hot? Environ Health Perspect 2010; 118: 80-6.

15. Kovats RS, Hajat S, Wilkinson P. Contrasting patterns of mortality and hospital admission during hot weather and heat waves in Great London, UK. Occup Environ Med 2004; 61: 893-8.

16. Feigin VL, Niktin YP, Bots ML, Vinogradova TE, Grobbee DE. A population-based study of the association of stroke occurrence with weather parameters in Siberia, Russia (1982-92). Eur J Neurol 2000; 7: 171-8.

17. Jiménez-Conde J, Ois A, Gomis M, Rodríguez-Campello A, Cuadrado-Godia E, Subirana I, et al. Weather as a trigger of stroke. Meteorological daily factors and incidence of stroke subtypes. Cerebrovasc Dis 2008; 26: 348-54.

18. Cowperthwaite MC, Burnett MG. An analysis of admission from 155 United States hospitals to determine the influence of weather on stroke incidence. J Clin Neurosci 2011; 18: 618-23.

19. Lago A, Geffner D, Tembl J, Landete I, Valero C, Baquero M.
Circadian variation in acute ischemic stroke: a hospital-based study. Stroke 1998; 29: 1873-5.

20. Jiménez-Conde J, Ois A, Rodríguez-Campello A, Gomis M, Roquer J. Does sleep protect against ischemic stroke? Less frequent ischemic strokes but more severe ones. J Neurol 2007; 254: 782-8

21. Jiménez-Conde J, Roquer J. Los ritmos del ictus isquémico: factores externos que contribuyen a modular el momento de aparición de los eventos. Med Clin (Barc) 2009; 132: 671-6.

22. Elliott WJ. Circadian variation in the timing of the stroke onset: a meta-analysis. Stroke 1998; 29: 992-6.

23. Serena J, Dávalos A, Segura T, Mostacero E, Castillo J. Stroke on awakening: looking for a more rational management. Cerebrovasc Dis 2003; 16: 128-33.

24. Segura T, Vega G, López S, Rubio F, Castillo J; on behalf of the Cerebrovascular Diseases Study Group of the Spanish Society of Neurology. Public perception of stroke in Spain. Cerebrovasc Dis 2003; 16: 21-6.

25. Castillo J. Discriminación neurovascular: ahora nosotros podemos ser el problema. Rev Neurol 2011; 52: 129-30.

26. Rothwell PM, Algra A, Amarenco P. Medical treatment in acute and long-term secondary prevention after transient ischaemic attack and ischaemic stroke. Lancet 2011; 377: 1681-92.

27. Crimmius DS, Levi CR, Gerraty RP, Beer CD, Hill KM; National Stroke Foundation Acute Stroke Guidelines Expert Working Group. Acute stroke and transient ischaemic attacks management. Time to act fast. Intern Med J 2009; 39: 325-31.

Hospital arrival times and delay before acute stroke patients receive neurological care. Analysis of a national multi-centre registry: EPICES registry (II)

Introduction. Delays on arrival time and specialized care at hospital are still the first cause that hampers the application of best possible treatments to the stroke patients.

Aim. To analyze the arrival time to the hospital up to the neurological care of patients, in a multicentric series of stroke patients.

Patients and methods. The EPICES registry is an observational and prospective study performed with a methodology of registering consecutive clinical records of stroke patients admitted to the hospital under neurological attention. The sample used to analyze starting-door times it was 5,454 patients, for the door-neurologist time it was 5,379 patients, and for the time of stay at ER room it was 5,825 patients.

Results. $90 \%$ of the patients presented a stroke between 8 and $24 \mathrm{~h}$. The season of the year had no influence on the frequency, neither on the distribution of stroke type. onset-door time was $264.1 \pm 318.3$ minutes; $34.7 \%$ of the patients arrived to the hospital during the first hour after the onset of the symptoms, and $63.7 \%$ of them arrived during the first three hours. Door-neurologist time was $378.1 \pm 731.4$ minutes; patients' age $(p<0.0001)$ and previous strokes $(p<0.0001)$ significantly prolonged such time. The availability of neurologist on call at the emergency service and of stroke units were associated to a significant reduction of hospital indoor times.

Conclusion. In relation to previous data, current study shows an important reduction of onset-door and door-neurologist times.

Key words. Assistance delay. Assistance facilities. Indoor care delay. Outdoor care delay. Stroke. 